\title{
Coordonner les mesures pour renforcer la sécurité des patients
}

\author{
Michael Wehrlia ${ }^{a}$ Thierry Fumeaux ${ }^{b}$, Hans Ulrich Rothen ${ }^{c}$, Martin Balmer ${ }^{d}$ \\ a Président exécutif de la SSMI; ${ }^{b} \operatorname{Pr}$ Dr méd., président des médecins de la SSMI, membre de la FMH; ${ }^{c} \operatorname{Pr}$ Dr méd., vice-président de la Commission \\ Données de la SSMI, lead de la Commission Qualité de la SSMI, membre de la FMH; d Assesseur du comité de la SSMI, président de la Commission Qualité \\ de la SSMI
}

\begin{abstract}
Garantir et améliorer la qualité des traitements et la sécurité des patients dans les unités de soins intensifs en Suisse comptent depuis longtemps parmi les missions principales de la Société Suisse de Médecine Intensive (SSMI). Depuis 1976, la Société a mis en place et applique des directives pour reconnaître les unités de soins intensifs du pays. Cette procédure de certification interprofessionnelle des unités de soins intensifs développée au fil des années sur la base de ce processus de reconnaissance reste à ce jour unique au monde.
\end{abstract}

\begin{abstract}
Les données minimales de la SSMI (MDSi) sont étroitement liées à la certification des unités de soins intensifs. Les MDSi définissent et répertorient certains indicateurs de l'organisation et de l'activité d'une unité de soins intensifs, comme par exemple le taux de survie des patientes et patients après un séjour en soins intensifs. Les données anonymisées permettent de comparer les unités de soins intensifs de toute la Suisse. En outre, il est possible, grâce à ces données, d'évaluer si une unité de soins intensifs peut être certifiée par la
\end{abstract}

Les données anonymisées permettent de comparer les unités de soins intensifs de toute la Suisse.

Commission Certification de la SSMI. La Commission Données de la SSMI, responsable des MDSi, est l'une des cinq commissions qui permettent aujourd'hui de contrôler et garantir la sécurité des patients au sein du département «Qualité» de notre société.

\section{Elargir ses propres horizons}

Malgré cette longue tradition et l'importance donnée à la sécurité des patients par notre société, il est bon d'analyser ce qui se passe ailleurs et d'écouter les expertes et experts d'autres disciplines et secteurs, dans le but de poursuivre l'effort d'amélioration de la sécurité des patients, grâce au processus d'assurance qualité. C'est la raison pour laquelle le symposium annuel de la SSMI, qui s'est tenu le 27 avril dernier, a servi de plateforme à un échange interprofessionnel et interdisciplinaire sur le thème de la sécurité des patients. Cette année, la SSMI a reçu le soutien de la FMH et de la fondation Sécurité des patients Suisse, qui ont assuré ensemble le patronat du symposium.

Pour améliorer la qualité, il est de coutume de commencer par analyser les erreurs. Comme le mentionne Bernhard Frey de l'Hôpital universitaire pédiatrique de Zurich, les erreurs de traitement médicamenteux peuvent déjà être diminuées en limitant le nombre de médicaments prescrits. L'an dernier, la SSMI a publié dans le cadre de l'initiative «Smarter Medicine» une liste de neuf mesures dont la prescription dans les unités de soins intensifs suisses devrait être reconsidérée, étant inutile pour la patiente ou le patient dans de nombreuses situations. Plusieurs de ces mesures concernent la prescription de médicaments.

Un étiquetage clair et univoque est une autre solution pour réduire le nombre d'erreurs de prescription: l'apparence extérieure d'un médicament doit se distinguer de celle de tout autre produit, et le dosage indiqué doit être clair et compréhensible. Et Bernhard Frey ajoute que, selon lui, l'utilisation d'un système électronique de gestion des données des patients (dossier électronique) permettrait de réduire davantage encore le nombre d'erreurs. Mais ce genre de système est loin d'être utilisé dans tous les hôpitaux. D'ici à ce que ce soit le cas, la mise en œuvre d'un autre système de contrôle peut contribuer à éviter les erreurs. Ainsi, à l'Hôpital universitaire pédiatrique de Zurich, un pharmacien hospitalier accompagne régulièrement les visites. 
Alison Lovatt et Ali Cracknell, qui ont introduit des huddles dans des services hospitaliers du Nord de l'Angleterre, confirment elles aussi que l'implication de tous les groupes professionnels et disciplines concernés renforce la culture de la sécurité. Lors de ces réunions courtes (cinq à quinze minutes), organisées tous les jours à la même heure et au même endroit, les participants discutent, debout de préférence, des situations

\section{Comme l'ensemble de l'équipe interprofes-} sionnelle est présent, la sécurité des patients se voit renforcée.

et des patientes et patients à risque. Comme l'ensemble de l'équipe interprofessionnelle est présent, la sécurité des patients se voit renforcée. C'est ce que confirme Michael Simon de l'Université de Bâle dans son allocution générale d'introduction. Selon Alison Lovatt et Ali Cracknell, les huddles permettent d'éviter efficacement des événements indésirables, ils améliorent la communication au sein de l'équipe et la sécurité des patients.

\section{Ensemble pour une sécurité accrue des patients}

En matière de qualité aussi, les collaborations entre différentes organisations et disciplines sont absolument nécessaires. En 2016, l'Académie suisse pour la qualité en médecine (ASQM) a publié une charte de qualité qui depuis a été signée par 69 organisations, parmi lesquelles la SSMI. Comme l'indique Christoph Bosshard, vice-président de la $\mathrm{FMH}$, cette promesse de qualité repose sur les trois piliers que sont la transparence, l'engagement et la durabilité. Les groupes signataires confèrent plus de visibilité à la qualité des services médicaux ainsi qu'aux activités médicales existantes et nouvelles qui encouragent la qualité en médecine. En signe de leur engagement, ils développent également une stratégie qualité et évaluent régulièrement les travaux par le biais d'un rapport qualité. Dans ce contexte, la SSMI est même allée plus loin en créant à l'automne dernier sa Commission Qualité interprofessionnelle, dont la première action a été de présenter sa propre charte qualité.

En outre, par leur déclaration d'intention, les organisations inscrivent la stratégie qualité dans un projet à plus long terme qui garantit le développement de la qualité. Par ailleurs, le travail réalisé en matière de qualité par toutes les organisations signataires de la charte fait partie des formations, formations postdiplômes et formations continues prodiguées au niveau médical.

\section{Apprendre des données}

Selon Bernard Burnand de l'Université de Lausanne, il est important de répertorier les événements indésirables. Mais dans ce contexte, il faut aussi que la documentation soit facile à mettre en œuvre. Des outils numériques peuvent s'avérer utiles. Jonas Marschall de l'Hôpital de l'Ile à Berne indique que dans le domaine des infections nosocomiales, Swissnoso, qui est le centre national pour la prévention des infections, offre différents modules et applications permettant de surveiller et d'empêcher à moindre coût et de manière pratique les infections nosocomiales, pour in fine accroître davantage la sécurité des patients.

\section{Des modifications structurelles plutôt que des mesures individuelles}

Dans son allocution sur le renforcement de la sécurité des patients passant par une meilleure conception, David Schwappach de la fondation Sécurité des patients Suisse indique que, souvent, on privilégie des interventions individualisées et moins lourdes là où des changements structurels profonds seraient en fait nécessaires pour améliorer effectivement la sécurité des patients. Ainsi, si des formations, des procédures standards (SOP) et des fiches sont élaborées ou mises en place facilement, elles sont toutefois souvent inutiles ou inutilisables dans la pratique. David Schwappach, dont l'allocution est consultable en web casting sur le site de la SSMI, illustre son propos en prenant l'exemple de la préparation des anesthésiants avant les opérations [1]. Pour éviter les erreurs de médication lors de l'anesthésie, des médecins ont développé en collaboration avec des concepteurs industriels un plateau pour seringues qui, grâce à des creux marqués,

\section{Pour éviter les erreurs de médication lors de} l'anesthésie, des médecins ont développé en collaboration avec des concepteurs industriels un plateau pour seringues.

empêche les seringues de glisser, et dont les codes couleurs assurent la bonne affectation de chaque médicament. De cette manière, les médecins, infirmières et infirmiers voient leur travail facilité, et ils peuvent se concentrer davantage sur la patiente ou le patient, avec un niveau de sécurité renforcé.

Pour Anthony Staines de la Fédération des Hôpitaux Vaudois (FHV), les mesures individuelles sont insuffisantes. Si l'on souhaite améliorer durablement la culture de la sécurité dans les unités de soins intensifs, il est nécessaire d'adopter une approche multi- 
modale basée sur une combinaison d'outils qui tous permettent de poursuivre un seul et même objectif. Ainsi, l'hygiène des mains a pu être renforcée dans di-

\section{$\mathrm{Au}$ lieu de se contenter d'analyser les erreurs pour contribuer à une sécurité renforcée des patients, il est nécessaire aussi d'examiner les exemples de réussites.}

vers hôpitaux du canton de Vaud grâce à l'utilisation, d'une part, de brochures, posters et autocollants indiquant l'importance d'une désinfection minutieuse et régulière, et par la mise à disposition, d'autre part, d'un nombre accru de distributeurs doseurs dans les unités.

\section{Changement culturel}

Sven Ständer de l'Hôpital de Männedorf appelle à un changement culturel fondamental. Au lieu de se contenter d'analyser les erreurs pour contribuer à une sécurité renforcée des patients, il est nécessaire aussi d'examiner les exemples de réussites. La démarche ne permettra pas d'éviter les échecs, mais elle garantira que tout se déroule correctement. Dans ce concept appelé «Safety 2.0», la gestion réactive de la qualité devient un sujet proactif. Certains employés sont utilisés comme ressources pour analyser la routine quotidienne et pour comprendre les rares dysfonctionnements.

En fin de symposium, tous les participants ont pu mesurer toute la complexité de mettre en place des actions durablement efficaces. L'amélioration de la sécurité des patients reste un défi de taille pour tous les groupes professionnels impliqués - des mesures et analyses systémiques en équipe doivent continuer d'accroître à l'avenir la culture de la sécurité dans les unités de soins intensifs suisses - la Commission Qualité de la SSMI travaille dans ce cadre à la mise en œuvre de sa stratégie.

\section{Référence}

1 Grigg EB, Martin LD, Ross FJ, et al. Assessing the Impact of the Anesthesia Medication Template on Medication Errors During Anesthesia: A Prospective Study. Anesthesia Analgesia. 2017;124:1617-25; doi: 10.1213/ANE.0000000000001823.

\section{Congrès annuel de la SSMI}

Le congrès annuel de la SSMI se tiendra du 19 au 21 septembre et proposera d'intéressants ateliers et interventions interprofessionnels à Interlaken, où nous serions heureux de vous accueillir. Vous trouverez davantage d'informations sur le congrès et sur la SSMI à I'adresse www.sgi-ssmi.ch 